\title{
Simultaneous Occurrence of Papillary Carcinoma and Medullary Carcinoma
}

\author{
Lyza Camille Gadong and Thelma Crisostomo
}

Section of Endocrinology, Diabetes and Metabolism, Department of Internal Medicine, Makati Medical Center, Philippines

\begin{abstract}
The cell origin, histopathologic features, and prognosis of medullary and papillary thyroid carcinoma are different and to have them occur simultaneously in a single patient is a rare occurrence.

This is a case of a 38-year-old female who presented with an enlarging anterior neck mass whose fine needle aspiration biopsy could not rule out a papillary lesion. Thus, she was advised to undergo total thyroidectomy, and her final histopath showed a simultaneous medullary and papillary thyroid carcinoma. Her initial serum calcitonin was elevated at 252 pg/ $\mathrm{ml}$, and it remained persistently elevated over the course of 7 months. A repeat ultrasound revealed solid nodules with coarse calcifications and enlarged lymph nodes at both submandibular regions. This warranted a repeat surgery with neck dissection with the finding of eight lymph nodes positive for metastatic carcinoma. On follow up after her second surgery, the calcitonin decreased to $42.70 \mathrm{pg} / \mathrm{ml}$.
\end{abstract}

Knowledge of this simultaneous occurrence of medullary thyroid carcinoma and papillary cancer is important for its prognostic implications and therapeutic plan.

Key words: thyroid cancer, papillary thyroid cancer, medullary thyroid cancer

\section{INTRODUCTION}

The cell origin, histopathologic features, and prognosis of medullary thyroid carcinoma and papillary thyroid carcinoma are different and to have them occur simultaneously in a single patient is a rare event. Data about the simultaneous occurrence of these tumors are based mainly on case reports and series.

\section{CASE}

This is a case of a 38-year-old female who has simultaneous medullary thyroid cancer in the left thyroid lobe and papillary thyroid cancer in the right thyroid lobe. She was diagnosed to have thyrotoxicosis at 18 years old presenting with palpitations and an anterior neck mass. She was maintained on an unrecalled dose of carbimazole and propanolol for 1 year which was eventually discontinued since her thyroid function test normalized, with noted decrease in size of her anterior neck mass.

Five years prior, she began noticing an enlarging anterior neck mass. There was no associated hoarseness of voice, dysphagia, weight loss, palpitations or difficulty of breathing. She consulted an endocrinologist and work up was done. Thyroid function test showed an elevated $\mathrm{TSH}$ at $11.84 \mathrm{uIU} / \mathrm{ml}$ and a normal FT4 at $15 \mathrm{uIU} / \mathrm{ml}$. Her ultrasound showed an enlarged left thyroid gland with a $2.38 \times 1.4 \times 1.69$ solid nodule with calcifications and minimal hypervascularity. Few coarse parenchymal calcifications are noted at the mid portion of the right lobe. She underwent fine needle aspiration biopsy (FNAB) of her left thyroid lobe with a result of an adenomatous goiter with concomitant lymphocytic thyroiditis. She was given Levothyroxine $50 \mathrm{mcg}$ daily with serial monitoring of her thyroid function test and ultrasound.

Repeat ultrasound two years later showed interval increase in the size of the previously noted solid nodule with calcifications in the left lobe, with new nodules noted on the right lobe. This warranted a repeat biopsy. Repeat ultrasound guided fine needle aspiration biopsy of the nodule on the right thyroid gland showed nodular hyperplasia but cannot rule out papillary lesion. While the FNAB of the nodule on the left showed nodular hyperplasia of left thyroid lobe. Thus, she underwent total thyroidectomy.

Microscopic examination of the isthmus and left lobe were consistent with medullary carcinoma, with the tumor size $6 \mathrm{~cm}$ in its greatest dimension. The right lobe showed papillary thyroid carcinoma, with the tumor size in greatest dimension $1.1 \mathrm{~cm}$, with no lymphovascular or perineural invasion (Figure 1). Immunohistochemistry report for the isthmus and left lobe were chromogranin positive, calcitonin positive supporting the diagnosis of medullary thyroid carcinoma. 

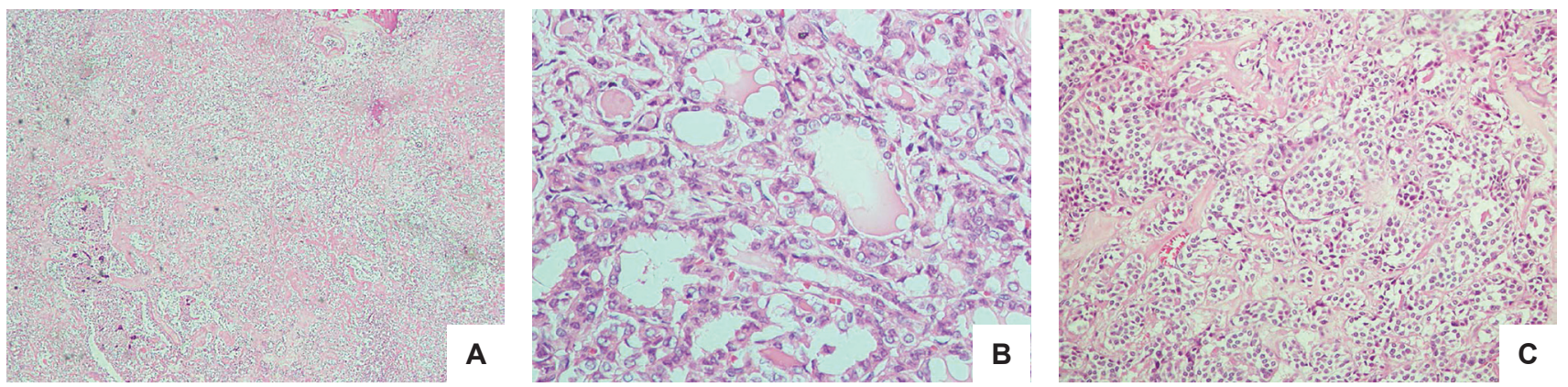

Figure 1. Histopath. (A) Areas of normal parenchyma, thyroiditis, and papillary carcinoma (H\&E, 40x). (B) Papillary Thyroid carcinoma, Right lobe. Overlapping clear ground glass nuclei with eosinophilic inclusions (H\&E, 400x). (C) Medullary carcinoma. Cells with eccentrically located round nuclei with salt and pepper nuclear chromatin pattern (H\&E, 100x).
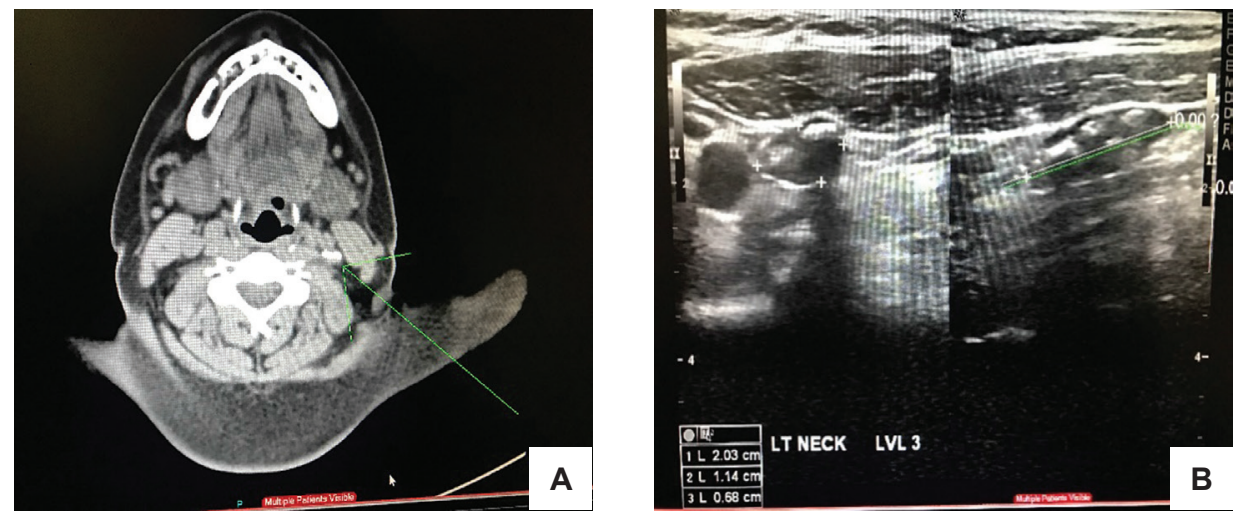

Figure 2. (A) CT scan of the neck with contrast. Calcification in the left infrahyoid carotid space possibly calcified lymph nodes. (B) Neck ultrasound. Nodes with calcifications in the left parajugular region (level 3).

After the surgery, serum thyroglobulin was $0.307 \mathrm{ng} / \mathrm{ml}$ with normal antithyroglobulin $90.859 \mathrm{IU} / \mathrm{ml}$, while serum calcitonin was elevated at $252 \mathrm{pg} / \mathrm{ml}$. The elevated serum calcitonin warranted a work up for possible metastasis. Thus, a neck ultrasound was requested which showed post thyroidectomy. Solid nodules with coarse calcifications, left parajugular region. Enlarged lymph nodes, both submandibular regions (Figure 2).

Findings on CT scan of the neck, chest and whole abdomen were nonspecific. CT scan of the neck revealed no evidence of cervical lymphadenopathy (Figure 2).

Chest CT scan showed nonspecific nodules along the left lung fissure and along the posterior hemithoraces. Whole abdomen CT scan showed mild hepatosplenomegaly, uterine myoma and Nabothian cyst. For further workup, a whole-body fluorodeoxyglucose PET scan was requested. Results showed no hypermetabolic malignant looking disease in the neck and elsewhere. The mildly hypermetabolic calcified left submandibular and left supraclavicular lymph nodes look inflammatory/reactive.

However, serum calcitonin on serial monitoring was persistently elevated $(362,197 \mathrm{pg} / \mathrm{ml})$ over the course of 7 months (Table 1). A repeat thyroid ultrasound showed solid nodules (4) with coarse calcifications at the left parajugular area (level 3).

Thus, she underwent neck exploratory dissection, with the finding of eight of twenty-five lymph nodes with metastatic carcinoma. On follow up three months after
Table 1. Laboratory Results

\begin{tabular}{lccc} 
& April 2017 & May 2017 & July 2017 \\
\hline Calcitonin $(0-11.5 \mathrm{pg} / \mathrm{ml})$ & 252 & 362 & 197 \\
Thyroglobulin $(2-70 \mathrm{ng} / \mathrm{ml})$ & 0.307 & & \\
Antithyroglobulin (up to $100 \mathrm{IU} / \mathrm{ml})$ & 90.859 & & \\
\hline
\end{tabular}

the operation, the calcitonin decreased to $42.70 \mathrm{pg} /$ $\mathrm{ml}$. Nature and prognosis of papillary and medullary thyroid carcinoma were explained to the patient, with plans of monitoring for recurrence. Medullary thyroid carcinoma can occur sporadically or it may be associated with hereditary syndromes such as Multiple Endocrine Neoplasia Type 2. Thus, molecular studies investigating RET germline mutations in exons 11, 15 and 16 were performed, and patient was found to be positive for a mutation in exon 15. She is now recommended for work up for pheochromocytoma and hyperparathyroidism to screen for multiple endocrine neoplasia 2 syndromes.

\section{DISCUSSION}

Papillary thyroid carcinoma (PTC) is the most common type of thyroid carcinoma $(90 \%)$, and it originates from the follicular cells of the endoderm. Medullary thyroid carcinoma, on the other hand, arises from the parafollicular C cells of the ultimobranchial body of the neural crest, and it represents only $5 \%$ of all thyroid cancers. ${ }^{1}$

The simultaneous occurrence of an independent medullary thyroid carcinoma, a tumor showing morphological features of medullary thyroid carcinoma (MTC) with 
positive immunoreactive calcitonin cells, along with papillary carcinomas in the same thyroid gland is uncommon, but it has been reported.

Although the simultaneous occurrence of papillary and medullary thyroid carcinoma in one patient may be entirely coincidental, several authors have tried to explain as to why it happens. According to Erhamamci (2014), the simultaneous occurrence of MTC and DTC in the same thyroid gland can occur in two ways: as a mixed tumor showing dual differentiation or as a collision tumor showing two separate different carcinomas. ${ }^{2}$ They concluded that out of 1420 patients, $0.28 \%$ of patients with DTC displayed simultaneous MTC. ${ }^{2}$ Machens (2011) followed 1019 patients diagnosed with papillary thyroid carcinoma, and found 26 patients had simultaneous MTC with a prevalence rate of $2.6 .^{3}$

Previous studies have also mentioned several other theories about this concurrence. The first is the 'stem cell theory' which describes a common stem cell that transform into both follicular and C-cell lineages. ${ }^{4}$ Another is the 'field effect theory' which proposes that simultaneous transformation of both follicular and C cells is a result of common neoplastic stimuli. On the other hand, the 'collision theory' suggests that two independent tumours are located in the same thyroid by simple coincidence. ${ }^{4}$

Papillary carcinoma generally has a good prognosis since most patients are diagnosed during the early stage of the disease (stage I or II), with $5 \%$ having a lethal outcome. ${ }^{5}$ Medullary thyroid carcinoma, on the other hand, may have an indolent clinical course or an aggressive variant with a high mortality rate. ${ }^{5}$ Distant metastases are present in $13 \%$ of patients at initial diagnosis and portend a poor prognosis, with a 10-year survival rate of only $40 \% .^{5}$ MTC is difficult to cure with a high recurrence rate of $50 \% .^{5}$ According to $\mathrm{WHO}$, the prognosis of mixed medullary and follicular thyroid carcinoma depends upon the medullary component. Thus, the presence of the medullary component makes the prognosis worse as compared to a pure papillary carcinoma. Patients who have clinically evident disease are best treated with a minimum of a total thyroidectomy and bilateral central neck dissection. ${ }^{5}$ The use of adjuvant radioiodine therapy for the papillary carcinoma component of this disease has been mentioned in several case reports with good results. In a study by Diogini et al. (2007), they presented a 65-year-old man with multicentric PTC and MTC associated with diffuse lymphocytic-type thyroiditis who underwent total thyroidectomy with neck dissection and subsequent radioiodine treatment. ${ }^{5}$ This patient had normal thyroglobulin and calcitonin levels on follow up. ${ }^{5}$

\section{CONCLUSION}

Knowledge of this simultaneous occurrence of medullary thyroid carcinoma and papillary cancer is important because of the poor prognosis associated with medullary thyroid carcinoma. This report also highlights the importance of immunohistochemical markers to make a correct diagnosis.

Ethical Considerations

Patient consent was obtained before submission of the manuscript.

Statement of Authorship

All authors certified fulfillment of ICMJE authorship criteria.

\section{Author Disclosure}

The authors declared no conflict of interest.

\section{Funding Source}

None.

\section{References}

1. Katoh H, Yamashita K, Enomoto T, Watanabe M. Classification and general considerations of thyroid cancer. Ann Clin Pathol. 2015;3:1045.

2. Erhamamci S, Reyhan M, Koçer NE, Nursal GN, Torun N, Yapar AF. Simultaneous occurrence of medullary and differentiated thyroid carcinomas. Report of 4 cases and brief review of the literature. Hell J Nucl Med. 2014;17(2):148-52. PMID: 24997082. https://doi. org/10.1967/s002449910137.

3. Machens A, Dralle H. Simultaneous medullary and papillary thyroid cancer: a novel entity? Ann Surg Oncol. 2012;19(1):37-44. PMID: 21626080. https://doi.org/10.1245/s10434-011-1795-z.

4. Adnan Z, Arad E, Dana J, Shendler Y, Baron E. Simultaneous occurrence of medullary and papillary thyroid microcarcinomas: A case series and review of the literature. J Med Case Rep. 2013;7:26. PMID: 23336429. PMCID: PMC3552861. https://doi.org/10.1186/17521947-7-26.

5. Dionigi G, Castano P, Bertolini V, et al. Simultaneous medullary and papillary thyroid cancer: two case reports. J Med Case Rep. 2007;1:133. PMID: 17997826. PMCID: PMC2194707. https://doi org/10.1186/1752-1947-1-133.

Authors are required to accomplish, sign and submit scanned copies of the JAFES Author Form consisting of: (1) Authorship Certification, that authors contributed substantially to the work, that the manuscript has been read and approved by all authors, and that the requirements for authorship have been met by each author; (2) the Author Declaration, that the article represents original material that is not being considered for publication or has not been published or accepted for publication elsewhere, that the article does not infringe or violate any copvrights or intellectual property rights, and that no references have been made to predatory/ suspected predatory journals; (3) the Author Contribution Disclosure, which lists the specific contributions of authors; and (4) the Author Publishing Agreement which retains author copyright, grants publishing and distribution rights to JAFES, and allows JAFES to apply and enforce an Attribution-Non-Commercial Creative Commons user license. Authors are also required to accomplish, sign, and submit the signed ICMJE form for Disclosure of Potential Conflicts of Interest. For original articles, authors are required to submit a scanned copy of the Ethics Review Approval of their research as well as registration in trial registries as appropriate. For manuscripts reporting data from studies involving animals, authors are required to submit a scanned copy of the Institutional Animal Care and Use Committee approval. For Case Reports or Series, and Images in Endocrinology, consent forms, are required for the publication of information about patients; otherwise, appropriate ethical clearance has been obtained from the institutional review board. Articles and any other material published in the JAFES represent the work of the author(s) and should not be construed to reflect the opinions of the Editors or the Publisher. 